\title{
A LANDMARK-BASED NONLINEAR ELASTICITY MODEL FOR MOUSE ATLAS REGISTRATION
}

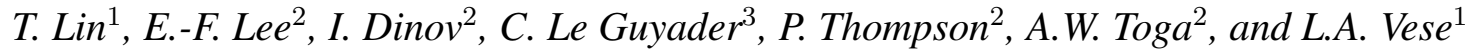 \\ ${ }^{1}$ Department of Mathematics, University of California, Los Angeles \\ ${ }^{2}$ Laboratory of Neuro Imaging, UCLA School of Medicine \\ ${ }^{3}$ Institute of Mathematical Research of Rennes, France
}

\begin{abstract}
This paper is devoted to the registration of gene expression data to a neuroanatomical mouse atlas in two dimensions. We use a nonlinear elasticity regularization allowing large deformations, guided by an intensity-based data fidelity term and by landmarks. We overcome the difficulty of minimizing the nonlinear elasticity functional by introducing an additional variable $\mathbf{v} \simeq \nabla \mathbf{u}$, where $\mathbf{u}$ is the displacement. Thus, in the obtained Euler-Lagrange equation, the nonlinearity is no longer in the derivatives of the unknown, $\mathbf{u}$. Experimental results show gene expression data mapped to a mouse atlas for a standard $L^{2}$ data fidelity term in the presence of landmarks. We also present comparisons with biharmonic regularization. An advantage of the proposed nonlinear elasticity model is that usually no regridding is necessary, while keeping the data term, regularization term and landmark term in a unified minimization approach.
\end{abstract}

Index Terms - mouse atlas, gene expression, registration, landmarks, nonlinear elasticity

\section{INTRODUCTION AND RELATED PRIOR WORK}

An important task in medical imaging, for clinical studies of disease and for atlas-based identification and segmentation of anatomical structures, is the comparison between a reference image $R$ (e.g. a labeled anatomical atlas) and a template image $T$ (e.g., an MRI scan). This is commonly done using image registration. Given a reference $R$ and a template $T$, both defined on the same image domain $\Omega$, we have to find a smooth invertible deformation $\Phi: \Omega \rightarrow \Omega$ so that the template is transformed into an image $T \circ \Phi$ "similar" to the reference.

An overview of mathematical methods for image registration is presented in [1] (including landmarks, $L^{2}$ similarity measure, splines, linear diffusion, linear elasticity, biharmonic and fluid regularizations). We also refer to related relevant work [2] for a well-known large deformation fluid reg-

This work was funded by the National Institutes of Health through the NIH Roadmap for Medical Research, Grant U54 RR021813 entitled Center for Computational Biology (CCB). istration method (not in variational form), and to a variational registration for large deformations (LDDMM) [3], [4].

A much related work, using non-linear elasticity regularization is [5], but by a different implementation based on the finite element method. We also refer to [6] for using nonlinear elasticity principles, but different from our proposed approach. Elasticity theory is also being used in [7], again implemented using the finite element method.

Different variational methods for regularization of the deformation (by linear elasticity or by diffusion tensor and geometry-driven regularization) using mutual information and other information-theoretic approaches, are presented in [8], in a theoretical framework.

We also wish to refer to related work [9], where a consistent landmark and intensity-based registration method is presented using thin-plate spline regularization (or biharmonic regularization). Another related reference where data, regularization and landmarks are combined, as in the present work, is [10]. We would like to mention the more recent work on a log-unbiased fluid registration method [11], [12].

We propose to use a nonlinear elasticity model for regularization of the displacement, since this allows smooth larger deformations without need of regridding most of the times. As we have mentioned, in prior work based on nonlinear elasticity principles, the finite element method has been used. However, we propose a different implementation by introducing an auxiliary variable for the Jacobian matrix of the displacement, that removes the nonlinearity in the derivatives (inspired from more theoretical work [13] and work [14] for a joint segmentation and registration model). We apply this idea to mapping gene expression data to mouse atlas.

Genetic mutations and knock-out strains of mice provide critical models for a variety of human diseases. Mouse brain atlases facilitate the integration of anatomic, genetic, and physiologic observations from multiple subjects in a common space. For example, the C57BL/6J mouse digital brain atlas [15], [16] is a comprehensive framework for storing and accessing information, and serves as a canonical representation of the mouse brain. Brain data alignment to an atlas is an important pre-processing step that ensures proper registration 
of imaging brain data to the atlas, usability of the atlas as a common and unbiased framework and facilitates statistical analysis and comparison of data from multiple subjects.

\section{DESCRIPTION OF THE MODELS}

We aim to compute a smooth deformation $\Phi(\mathbf{x})=\mathbf{x}+\mathbf{u}(\mathbf{x})$, by estimating the displacement vector field $\mathbf{u}$ from an energy minimization based on three terms: intensity similarity measure, regularization, and landmark-based distance function. Thus we solve the minimization problem

$$
\inf _{\mathbf{u}}\left\{J(\mathbf{u})=\operatorname{Fid}(\mathbf{u})+\alpha \operatorname{Reg}(\mathbf{u})+\gamma D^{L M}(\mathbf{u})\right\},
$$

by gradient descent in $\mathbf{u}=\left(u_{1}, u_{2}\right)$ in two dimensions:

$$
\frac{\partial u_{l}}{\partial t}=-\frac{\partial F i d}{\partial u_{l}}(\mathbf{u})-\alpha \frac{\partial R e g}{\partial u_{l}}(\mathbf{u})-\gamma \frac{\partial D^{L M}}{\partial u_{l}}(\mathbf{u}), l=1,2 .
$$

\subsection{Similarity measure}

We chose the standard $L^{2}$ distance as similarity measure between $T(\Phi)$ and $R$, and this is complemented by the use of additional landmarks as geometrical constraints. We will see that, even if $T$ (gene data) and $R$ (mouse atlas) are of different modalities, satisfactory results are obtained without requiring more sophisticated distances, such as the mutual information. Therefore our fidelity term (similarity measure) is simply:

$$
\begin{aligned}
& F i d(\mathbf{u})=\frac{1}{2} \int_{\Omega}|T(\mathbf{x}+\mathbf{u}(\mathbf{x}))-R(\mathbf{x})|^{2} d \mathbf{x}, \text { with } \\
& \frac{\partial F i d}{\partial u_{l}}(\mathbf{u})=(T(\mathbf{x}+\mathbf{u}(\mathbf{x}))-R(\mathbf{x})) \frac{\partial T}{\partial x_{l}}(\mathbf{x}+\mathbf{u}(\mathbf{x})) .
\end{aligned}
$$

\subsection{Landmark distance function}

The landmark distance $D^{L M}$ can be defined as a quadratic penalty term as follows [1]:

$$
\begin{array}{r}
D^{L M}(\mathbf{u})=\frac{1}{2} \sum_{k=1}^{m}\left\|\mathbf{x}^{R, k}-\Phi\left(\mathbf{x}^{T, k}\right)\right\|^{2}=\frac{1}{2} \sum_{k=1}^{m}\left[\left(x_{1}^{R, k}\right.\right. \\
\left.\left.-\left(x_{1}^{T, k}+u_{1}\left(\mathbf{x}^{T, k}\right)\right)\right)^{2}+\left(x_{2}^{R, k}-\left(x_{2}^{T, k}+u_{2}\left(\mathbf{x}^{T, k}\right)\right)\right)^{2}\right],
\end{array}
$$

where $\|\cdot\|$ denotes the Euclidean norm, $\mathbf{x}^{R, k}$ and $\mathbf{x}^{T, k}$ are the landmarks chosen from the reference image and template image respectively, and $\Phi\left(\mathbf{x}^{T, k}\right)=\mathbf{x}^{T, k}+\mathbf{u}\left(\mathbf{x}^{T, k}\right)$. The geometrical constraint is enforced by making $\gamma>0$ progressively larger with time. For the gradient descent, we obtain

$$
\frac{\partial D^{L M}}{\partial \mathbf{u}}(\mathbf{u})(\mathbf{x})=-\left\{\begin{array}{l}
0 \text { if } \mathbf{x} \neq \mathbf{x}^{T, k} \\
\mathbf{x}^{R, k}-\mathbf{x}^{T, k}-\mathbf{u}\left(\mathbf{x}^{T, k}\right)
\end{array}, k=1, . ., m .\right.
$$

\subsection{Regularization}

Our main choice of the regularization term is inspired from nonlinear elasticity, since, by comparison with linear diffusion, linear elasticity or biharmonic regularizations, it may allow larger deformations without regridding. We propose a particular implementation that removes the non-linearity in the derivatives, inspired by [13], [14]. In our experimental results, we will compare the non-linear elasticity regularization with the more standard $\dot{H}^{2}$ regularization or biharmonic regularization (called curvature regularization in J. Modersitzki [1]).

\subsubsection{Nonlinear Elasticity Regularization}

Assuming that the material undergoes large deformations, the strain energy is given by (corresponding to Saint VenantKirchhoff hyperelastic materials) [17] $\operatorname{Reg}(\mathbf{u})=\int_{\Omega} W(\epsilon) d \mathbf{x}$, with tensor $\epsilon(\mathbf{u})=\frac{1}{2}\left(\nabla \mathbf{u}^{t}+\nabla \mathbf{u}+\nabla \mathbf{u}^{t} \nabla \mathbf{u}\right)$ and stored energy

$$
\begin{array}{r}
W(\epsilon)=\frac{\lambda}{2}(\operatorname{trace}(\epsilon))^{2}+\mu \operatorname{trace}\left(\epsilon^{2}\right)=\frac{\lambda}{8}(2(\operatorname{div} \mathbf{u}) \\
\left.+\sum_{k=1}^{2}\left|\nabla u_{k}\right|^{2}\right)^{2}+\frac{\mu}{4}\left(\sum_{i=1}^{2}\left[2 \frac{\partial u_{i}}{\partial x_{i}}+\sum_{k=1}^{2}\left(\frac{\partial u_{k}}{\partial x_{i}}\right)^{2}\right]^{2}\right. \\
\left.+\sum_{i, j=1, i \neq j}^{2}\left[\frac{\partial u_{j}}{\partial x_{i}}+\frac{\partial u_{i}}{\partial x_{j}}+\sum_{k=1}^{2} \frac{\partial u_{k}}{\partial x_{i}} \frac{\partial u_{k}}{\partial x_{j}}\right]^{2}\right) .
\end{array}
$$

Here $\lambda$ and $\mu$ are the Lamé coefficients of the material. An admissible deformation field $\Phi: \bar{\Omega} \rightarrow \bar{\Omega}, \Phi(\mathbf{x})=\mathbf{x}+\mathbf{u}(\mathbf{x})$, should satisfy $\operatorname{det} \nabla \Phi>0$ in $\Omega, \Phi(\mathbf{x})=\mathbf{x}$ on $\partial \Omega$, and $\Phi$ is one-to-one and onto on $\bar{\Omega}$. If the nonlinear term above $\nabla \mathbf{u}^{t} \nabla \mathbf{u}$ were dropped, then this would correspond to the linear elasticity assumption, appropriate for small deformations.

It is cumbersome to directly compute and discretize the associated Euler-Lagrange equation in $\mathbf{u}$. To avoid this difficulty, we introduce in two dimensions the variable $\mathbf{v}=\left(\begin{array}{ll}v_{11} & v_{12} \\ v_{21} & v_{22}\end{array}\right)$, which approximates $\nabla \mathbf{u}$. For $\beta$ large enough, $\operatorname{Reg}(\mathbf{u})$ can be well approximated by

$$
\begin{array}{r}
\operatorname{Reg}_{\beta}(\mathbf{u}, \mathbf{v})=\int_{\Omega}\left[W\left(\frac{1}{2}\left(\mathbf{v}^{t}+\mathbf{v}+\mathbf{v}^{t} \mathbf{v}\right)\right)+\beta|\mathbf{v}-\nabla \mathbf{u}|^{2}\right] d \mathbf{x} \\
=\int_{\Omega}\left(\frac{\lambda}{8}\left[2\left(v_{11}+v_{22}\right)+\left(v_{11}^{2}+v_{12}^{2}+v_{21}^{2}+v_{22}^{2}\right)\right]^{2}\right. \\
+\frac{\mu}{4}\left[\left(2 v_{11}+v_{11}^{2}+v_{21}^{2}\right)^{2}+\left(2 v_{22}+v_{12}^{2}+v_{22}^{2}\right)^{2}\right. \\
\left.\left.+2\left(v_{12}+v_{21}+v_{11} v_{12}+v_{21} v_{22}\right)^{2}\right]\right) d \mathbf{x} \\
+\beta \int_{\Omega}\left[\left|v_{11}-\frac{\partial u_{1}}{\partial x_{1}}\right|^{2}+\left|v_{12}-\frac{\partial u_{1}}{\partial x_{2}}\right|^{2}\right. \\
\left.+\left|v_{21}-\frac{\partial u_{2}}{\partial x_{1}}\right|^{2}+\left|v_{22}-\frac{\partial u_{2}}{\partial x_{2}}\right|^{2}\right] d \mathbf{x} .
\end{array}
$$


The (linear) Euler-Lagrange equation in $u_{l}, l=1,2$ is

$$
\frac{\partial \operatorname{Reg}_{\beta}(\mathbf{u}, \mathbf{v})}{\partial u_{l}}=-2 \beta\left(\triangle u_{l}-\frac{\partial v_{l 1}}{\partial x_{1}}-\frac{\partial v_{l 2}}{\partial x_{2}}\right) .
$$

The Euler-Lagrange equation in $\mathbf{v}$ by gradient descent is

$$
\begin{aligned}
\frac{\partial v_{11}}{\partial t} & =2 \alpha \beta\left(\frac{\partial u_{1}}{\partial x_{1}}-v_{11}\right)-\alpha \lambda I\left(1+v_{11}\right) \\
& -\alpha \mu\left(2 v_{11}+v_{11}^{2}+v_{21}^{2}\right)\left(1+v_{11}\right)-\alpha \mu I I v_{12} \\
\frac{\partial v_{12}}{\partial t} & =2 \alpha \beta\left(\frac{\partial u_{1}}{\partial x_{2}}-v_{12}\right)-\alpha \lambda I v_{12} \\
& -\alpha \mu\left(2 v_{22}+v_{12}^{2}+v_{22}^{2}\right) v_{12}-\alpha \mu I I\left(1+v_{11}\right) \\
\frac{\partial v_{21}}{\partial t} & =2 \alpha \beta\left(\frac{\partial u_{2}}{\partial x_{1}}-v_{21}\right)-\alpha \lambda I v_{21} \\
& -\alpha \mu\left(2 v_{11}+v_{11}^{2}+v_{21}^{2}\right) v_{21}-\alpha \mu I I\left(1+v_{22}\right) \\
\frac{\partial v_{22}}{\partial t} & =2 \alpha \beta\left(\frac{\partial u_{2}}{\partial x_{2}}-v_{22}\right)-\alpha \lambda I\left(1+v_{22}\right) \\
& -\alpha \mu\left(2 v_{22}+v_{12}^{2}+v_{22}^{2}\right)\left(1+v_{22}\right)-\alpha \mu I I v_{21}
\end{aligned}
$$

where $I=v_{11}+v_{22}+\frac{1}{2} v_{11}^{2}+\frac{1}{2} v_{21}^{2}+\frac{1}{2} v_{12}^{2}+\frac{1}{2} v_{22}^{2}$, and $I I=v_{12}+v_{21}+v_{11} v_{12}+v_{21} v_{22}$.

\subsubsection{Biharmonic Regularization}

The $\dot{H}^{2}$ (biharmonic) regularization is

$$
\operatorname{Reg}(\mathbf{u})=\frac{1}{2} \sum_{l=1}^{2} \int_{\Omega}\left(\triangle u_{l}\right)^{2} d \mathbf{x}, \text { with } \frac{\partial \operatorname{Reg}}{\partial \mathbf{u}}(\mathbf{u})=\triangle^{2} \mathbf{u} .
$$

\section{EXPERIMENTAL RESULTS}

We show experimental results obtained by the two methods presented in the previous section for mapping one 2D slice of gene expression data (template $T$ ) to a $2 \mathrm{D}$ slice of the mouse atlas (reference $R$ ), in the presence of landmarks. The data is provided by the Center for Computational Biology, UCLA. The methods have been independently tested on 8 pairs, all of size 200x200 pixels, with the mouse brain and the gene expression located in the center of the image, over a black background (thus the non-brain regions have been removed, to produce better matching). The number of iterations for both methods depends on how small we wish the landmark distance and the similarity measure to be. The landmarks are marked by an experienced neuroanatomist based on the anatomical structures present in the images. This is based on prior knowledge in neuroanatomy. A position probability model might be derived, function of local geometric features. However, in real cases, methods for preparing gene expression images and results are very different. We do not know what would be the appropriate approach to derive a probability model that would reflect such diversity. The mouse atlas acquired from the LONI database was pre-segmented. The gene expression data was segmented manually to facilitate data processing in other applications. Some studies have developed algorithms for automatically segmenting the brain area of gene expression data.

Fig. 1 shows an example of such a data pair. We show the deformation grid with positions of landmarks superimposed (Fig. 2), and a color map of the Jacobian values given by $\operatorname{det}(\nabla \Phi)$ in Fig. 3, for both biharmonic and nonlinear elasticity regularization. No regridding was necessary, and the Jacobian values remain strictly positive. We notice that the nonlinear elasticity regularization produces better landmark matching; also, the more deformed regions have undergone expansion, while using the biharmonic regularization, the more deformed regions have undergone contraction. Similar results have been obtained for other mouse gene-atlas pairs. More tests have to be done.
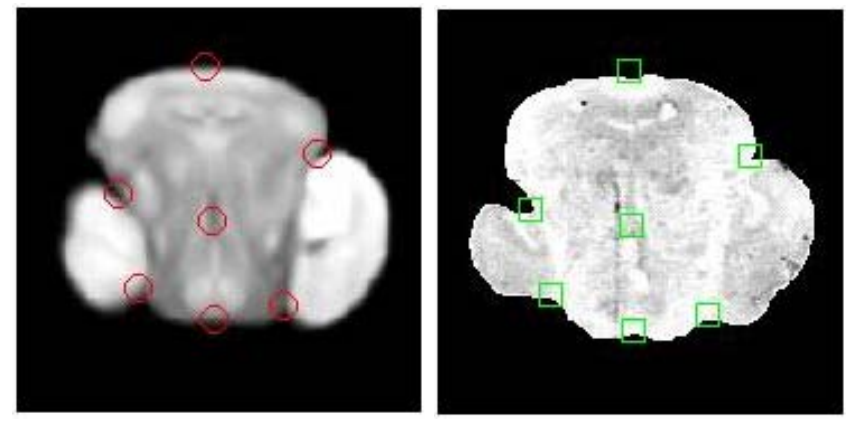

Fig. 1. Mouse atlas (reference $R$, left) and gene expression (template $T$, right) with specified landmarks.

\section{CONCLUSION AND FUTURE WORK}

We presented variational registration models for obtaining smooth deformations between two dimensional slices of mouse atlas and gene expression data. We proposed a nonlinear elastic regularization with an implementation that removes the nonlinearity in the derivatives. Experimental results were shown that required relatively large deformations and enforced landmark constraints, but no regridding was necessary. A better parameter selection and extension to three dimensions will be made.

\section{REFERENCES}

[1] J. Modersitzki, Numerical Methods for Image Registration, Oxford University Press, 2004.

[2] G.E. Christensen, R.D. Rabbitt, and M.I. Miller, "Deformable templates using large deformation kinematics," IEEE TIP, vol. 5, no. 10, pp. 1435-1447, 1996.

[3] F. Beg, M. Miller, A. Trouvé, and L. Younes, "Computing large deformation metric mappings via geodesic flows of diffeomorphisms," IJCV, vol. 61, no. 2, pp. 139-157, 2005. 

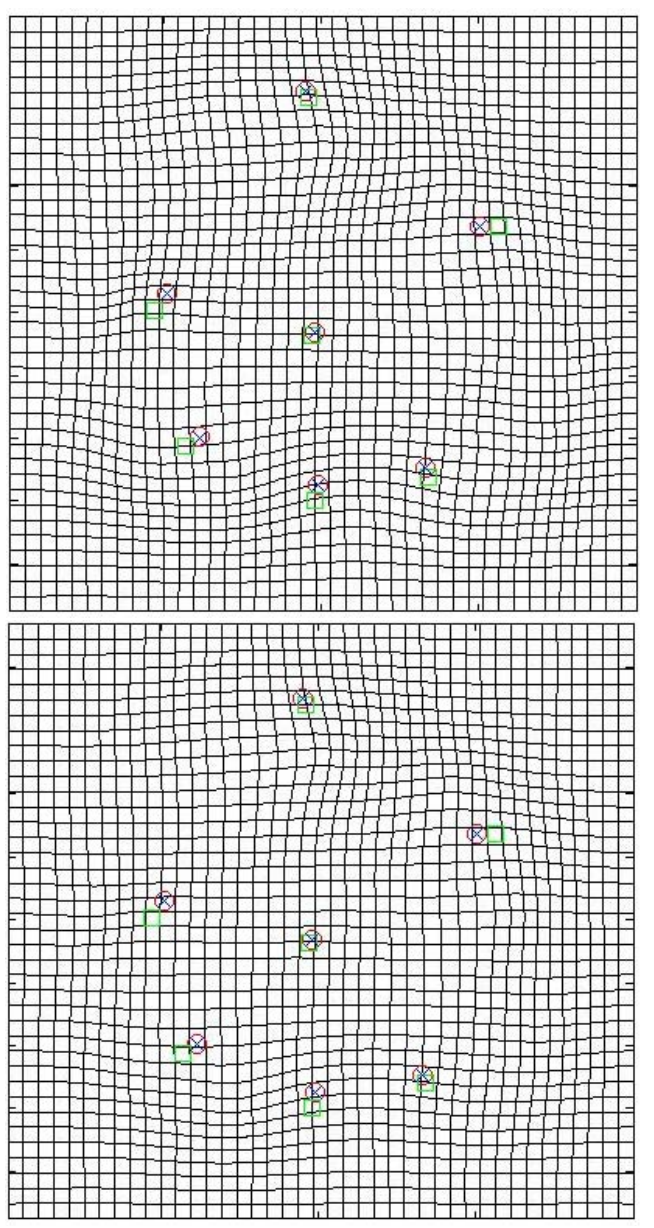

Fig. 2. Deformed grid using biharmonic (top) and nonlinear elasticity (bottom) regularizations (red ०: target landmarks; green $\square$ : source landmarks; blue $\times$ : transformed landmarks).

[4] M.I. Miller, A. Trouvé, and L. Younes, "On the metrics and Euler-Lagrange equations of computational anatomy," Аnnu. Rev. B. Eng, vol. 4, pp. 375-405, 2002.

[5] R. D. Rabbitt, J. A. Weiss, G. E. Christensen, and M. I. Miller, "Mapping of hyperelastic deformable templates using the finite element method," in Proceedings SPIE, 1995, vol. 2573, pp. 252-265.

[6] M. Droske and M. Rumpf, "A variational approach to non-rigid morphological registration," SIAM Appl. Math., vol. 64, no. 2, pp. 668-687, 2004.

[7] W. Peckar, C. Schnörr, K. Rohr, and H.S. Stiehl, "Parameter-free elastic deformation approach for 2D and 3D registration using prescribed displacements," JMIV, vol. 10, no. 2, pp. 143-162, 1999.

[8] O. Faugeras and G. Hermosillo, "Well-posedness of two nonrigid multimodal image registration methods," SIAM Appl. Maths., vol. 64, no. 5, pp. 1550-1587, 2004.
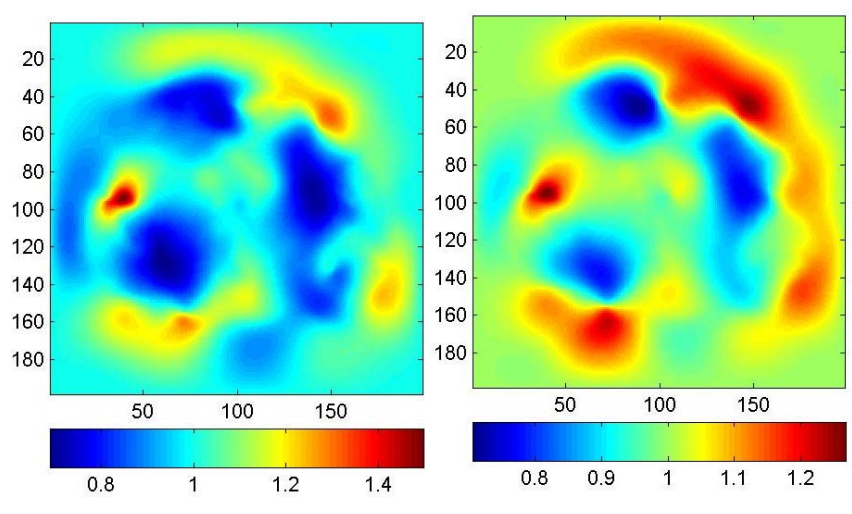

Fig. 3. Jacobian values using biharmonic (left) and nonlinear elasticity (right) regularizations.

[9] H.J. Johnson and G.E. Christensen, "Consistent landmark and intensity-based image registration," IEEE TMI, vol. 21, no. 5, pp. 450-461, 2002.

[10] C.O.S. Sorzano, P. Thévenaz, and M. Unser, "Elastic registration of biological images using vector-spline regularization," IEEE T. Biom. Eng., vol. 52, no. 4, pp. 652-663, 2005.

[11] I. Yanovsky, S. Osher, P. Thompson, and A. Leow, "Log-unbiased large-deformation image registration," VISAPP, vol. 1, pp. 272-279, 2007.

[12] I. Yanovsky, P. Thompson S. Osher, and A. Leow, "Topology preserving log-unbiased nonlinear image registration: Theory and implementation," $C V P R, \mathrm{pp}$. 1-8, 2007.

[13] P.V. Negrón Marrero, “A numerical method for detecting singular minimizers of multidimensional problems in nonlinear elasticity," Numerische Mathematik, vol. 58, no. 1, pp. 135-144, 1990.

[14] C. Le Guyader and L.A. Vese, "A combined segmentation and registration framework with a nonlinear elasticity smoother," UCLA CAM Report 08-16, March 2008.

[15] A.J. MacKenzie-Graham, E.-F. Lee, I.D. Dinov, H. Yuan, R.E. Jacobs, and A.W. Toga, "Multimodal, multidimensional models of mouse brain," Epilepsia, vol. 48, Supplement 4, pp. 75-81(7), 2007.

[16] E.-F. Lee, R.E. Jacobs, I. Dinov, A. Leow A, and A.W. Toga, "Standard atlas space for c57bl/6j neonatal mouse brain," Anat. Embryol (Berl)., vol. 210, no. 4, pp. 245263, 2005.

[17] R.M. Temam and A.M. Miranville, Mathematical Modeling in Continuum Mechanics, Cambridge, 2005. 